\title{
THE EVOLUTIONARY CODE CESAM: NUMERICAL TECHNIQUES
}

\author{
P. MOREL \\ Cassini, URA CNRS 1362, Observatoire de la Côte d'Azur, Nice
}

\begin{abstract}
CESAM is a consistent set of programs and routines designed for the calculations of stellar evolution. Untill now it allows the computation of the evolution from PMS or ZAMS to helium flash for stellar masses of some solar mass. It is constructed in such a way that all the physics works as external routines. The numerical techniques are based on the B-spline formalism. This formalism used both for the integration of the differential equations and for $1 D$ and 2D interpolation schemes of various tables of physical data.
\end{abstract}

\section{I - OVERALL STRUCTURE}

CESAM has been implemented by many contributors, get together in the CNRS GDR 131. The set of partial differential equations of stellar evolution is split as usual into two ordinary differential problems: the quasi-static model, a two-point boundary values problem, and an initial value problem, the evolution of the chemical composition in time. At each time step they are solved in turn until convergence.

CESAM treats the core and the envelope as a whole system, thus avoiding the usual matching, at some level, of a core and an envelope solutions obtained with different algorithms. The outer boundary conditions of the internal structure are implemented in the $P(R, L), T(R, L), M(R, L)$ form, for respectively the pressure, the temperature and the mass, $R$ and $L$ being the external radius and luminosity of the envelope; these conditions are applied deep enough in the atmosphere so that the medium is already optically thick.

\section{II - STATIC MODEL: B-SPLINE/COLLOCATION TECHNIQUE}

The equations of the two points value problem are solved using the spline/collocation technique. In this integration method, originated by De Boor (1978), the coordinates of each unknown $f_{j}, 1 \leq j \leq n_{e}$, of a sytem of $n_{e}$ ordinary differential equations of order 1 are projected as $f_{j}(x)=\sum_{i=1}^{M} f_{i, j} N_{i}^{m}(x)$ on the B-spline basis $\left\{N_{i}^{m}\right\}_{i=1}^{M}$ of order $m$, on a nodal vector - to be precised - constructed on the mesh points $x_{1}, x_{2}, \ldots, x_{n}$. Then the derivatives become $f_{j}^{\prime}(x)=\sum_{i=1}^{M} f_{i, j} \frac{d N_{i}^{m}(x)}{d x}$ and one has similar equations for the boundary conditions. The new unknowns, the reals $f_{i, j}$, are derived by writting that the equations are satisfied at an ad hoc number of so-called collocation points which are not necessarily the mesh points, as in finite difference methods. The basis beeing local, one has to deal with a block diagonal jacobian matrix for solving, with the Newton-Raphson method, the implicit set of stellar structure differential equations.

Moreover, as in Gaussian integration, with a judicious choice for the nodal vector and for the collocation points there is "superconvergency" at the mesh points where the order of precision of the solution is higher than the order of the B-splines. An another advantage of the method is the ability of retrieving exactly the numerical solution at any point simply by using the projections on the B-splines. That proves very convenient in 
the calculation of acoustic eigenmodes.

With the B-spline formalism it is possible to deal with discontinuities without too much difficulties, that simplify the calculations with convective mixing.

During the time evolution, according to the changes of the dependent variables, an automatic mesh refinement scheme optimizes the grid by insuring constant jumps from a mesh point to the next of a so-called "mesh distribution function" (Press et al., 1986).

Following Eggleton(1971) the set of dependent variables: $\ln P, \ln T, R^{2}, L^{2 / 3}$, with respect to $M^{2 / 3}$ avoids the central singularity of the equations of stellar structure and it can be shown (Morel, 1992) that it also provides an optimal numerical accuracy. On the other hand, the $2 / 3$ power of the luminosity - which avoids a singularity of the derivative of a square root on the vicinity of the origin - is not adapted to negative values of $L$. With CESAM the less accurate set of dependent variables: $\ln P, \ln T, M$, $L$ with respect to $R$ can be also used; there is no central singularity but an external free limit which is managed without difficulty by the automatic mesh point allocation scheme.

For the core and the envelope (calculated as a whole) a fixed number $n=300$ of grid points is usually used with B-splines of order $m=2$ that corresponds to $599=300 \times 2-1$ collocation points, hence the solution is of fourth-order accuracy for the quasi-static model. For the Sun, calculations have shown that higher number of mesh points or higher order for the B-spline does not lead to significant improvement of the internal accuracy (see Table I).

Many performing algorithms used in CESAM for B-spline calculations are taken from Schumaker(1981).

The three functions $P(R, L), T(R, L), M(R, L)$ which serve as outer boundary conditions are deduced from an atmosphere model, together with the derivatives which are needed for the iterative Newton-Raphson resolution of the implicit equations. This atmosphere model is integrated separately from the interior stratification, again with the B-spline/collocation procedure for arbitrary given $T(\tau)$ laws.

\section{III - EVOLUTION IN TIME OF THE CHEMICAL COMPOSITION}

The stiffy stable implicit BDF scheme (Hairer and Wanner 1991) is employed for the numerical integration of the set of differential equations governing the time evolution of the chemical species. The implicit equations are solved with the iterative NewtonRaphson algorithm. The choice of this scheme is guided by its properties of stiff-stability, and also because it is strictly conservative for all linear sets of the abundances. To ensure sufficient precision, one limits the changes of the chemical species during a time step. Only formula with accuracy order up to 2 are used. Due to the presence of the convective mixing the higher order schemes are almost useless.

In convective zones one assumes that the time scale of the turbulent eddies is less than the nuclear time scale, hence the time integration is performed on the mixed abundances time derivatives. A discontinuity is set on chemical composition distribution at each limit of growing convective zones. The B-spline formalism is also used for the interpolation of the chemical composition.

\section{IV - INTERPOLATION TECHNIQUES AND ACCURACY}

Without a significant loss of accuracy, tabulations and interpolations of physical data avoid important amount of calculations and allow an improvement of the numerical stability. Interpolation programs and routines with Lagrange, B-spline and Hermite schemes in $1 \mathrm{D}$ and $2 \mathrm{D}$ have been developped consistently with the numerical techniques already outlined. CESAM uses a $2 \mathrm{D}$ tensorial B-spline interpolation either in $(\ln T, \ln \rho)\left(\mathrm{LAOL}\right.$ tables) or in $\left(T_{6}, \ln \rho / T_{6}\right)$ (OPAL tables) and a $1 \mathrm{D}$ Lagrange scheme 
in chemical composition for the opacities, a 2D B-spline or Hermite scheme with respect to $(\ln P, \ln T)$ and a $1 \mathrm{D}$ five order polynomial fit at Chebychev points with respect to the chemical composition for the equation of state, a $1 \mathrm{D} \mathrm{B-spline} \mathrm{piecewise} \mathrm{polynomial}$ scheme for the thermonuclear reactions rates. One advantage of interpolation with the B-spline formalism is the control of the order of accuracy by only one parameter but convenient dual basis are not available as it does for Hermite and Lagrange interpolations.

TABLEI

\begin{tabular}{|c|c|c|c|c|c|c|}
\hline$n$ & $m$ & $\bar{X}$ & $\alpha$ & $\rho_{c}\left(\mathrm{~g} \mathrm{~cm}^{-3}\right)$ & $\mathrm{T}_{c}\left(10^{6} \mathrm{~K}\right)$ & $\mathrm{X}_{c}$ \\
\hline 300 & 2 & 0.70617 & 1.8982 & 152.676 & 15.5492 & 0.34233 \\
\hline & & $\overline{\Delta \mathrm{X} / \mathrm{X}}$ & $\overline{\Delta \alpha / \alpha}$ & $\overline{\Delta \rho_{c} / \rho_{c}}$ & $\overline{\Delta \mathrm{T}_{c}} / \mathrm{T}_{c}$ & $\Delta \mathrm{X}_{c} / \mathrm{X}_{c}$ \\
\hline 150 & 3 & 1. $10^{-6}$ & $7.710^{-5}$ & $7.910^{-6}$ & $-3.210^{-5}$ & $1.310^{-4}$ \\
\hline 300 & 3 & 1. $10^{-6}$ & $7.710^{-5}$ & $6.7 \quad 10^{-6}$ & $-1.10^{-6}$ & 6. $10^{-6}$ \\
\hline 600 & 3 & $2.410^{-5}$ & $-3.210^{-6}$ & $0.410^{-6}$ & $2.310^{-5}$ & 2. $10^{-4}$ \\
\hline
\end{tabular}

The accuracy of CESAM have been only investigated for calibrated Solar models. Internal accuracy is estimated from differences between models calculated with different number of shells $n$ and with different orders of B-spline $m$. A comparison of these models is given in Table I where the first line gives the initial hydrogen abundance, the mixing length, the central density, temperature and hydrogen abundance for calibrated models with $n=300$ and $m=2$. The other lines give the relative difference of the same quantities for the other models. Solar models computed by CESAM with simplified but fixed physics have been submitted to the SMC comparison organized by GONG, with respect to the other models submitted they present relative differences smaller than $10^{-4}$ (Christensen Dalsgaard, 1991; Gabriel, 1991).

A more detailed discussion of the accuracy for more realistic solar models is given in Morel et al. $(1990,1992)$.

\section{ACKNOWLEDGMENTS}

I am indebted to my collegues Drs. G. Berthomieu and J. Provost for stimulating discussions and suggesting improvements in the manuscript.

\section{REFERENCES}

Christensen-Dalsgaard J. 1991, Challenges to Theories of the Structure of Mode rate-Mass Stars, D.O. Gough, J. Toomre (Eds), Springer Verlag, p 11-36

De Boor C. 1978, A Practical Guide to Splines (Springer; third ed. 1985)

Eggleton, P. 1971 Mon. Not. R. astr. Soc. 151 351-364

Gabriel M. 1991, Challenges to Theories of the Structure of Mode rate-Mass Stars, D.O. Gough, J. Toomre (Eds), Springer Verlag, p 51-55

Hairer E., Wanner G. 1991, Solving ordinary differential equations II. Springler Verlag Morel P., Provost J., Berthomieu G. 1990, Solar Physics 128, 7

Morel P.,Berthomieu G., Provost J.,Lebreton Y. 1992, these proceedings

Morel P., 1992 in preparation

Press W.H., Flannery B.P., Teukolsky S.A., Vetterling W.T. 1986 Numerical Receipes, Cambridge University Press, Cambridge

Schumaker L. 1981, Splines Functions : Basic Theory, John Whiley. 